\title{
Fiabilidad y validez factorial del TCI-R en una muestra de adictos en tratamiento
}

\author{
Eduardo J. Pedrero-Pérez*
}

CAD 4. Instituto de Adicciones. Ayuntamiento de Madrid

\begin{abstract}
Resumen: El TCI-R es el más reciente instrumento de medida de la teoría biosocial de Cloninger y se ha mostrado como uno de los cuestionarios más útiles en la predicción de la conducta, especialmente en sus variantes patológicas. A pesar de que la versión española fue publicada hace siete años apenas contamos con estudios que avalen su bondad psicométrica. Se requiere conocer la fiabilidad y validez interna para su aplicación clínica. Se reclutó una muestra de 824 participantes que inician tratamiento por su adicción en un centro específico. Se realizaron pruebas de consistencia interna sobre los ítems, las subescalas y las dimensiones principales del cuestionario y se estudió la validez de constructo mediante análisis factoriales exploratorio multivariado, paralelo y confirmatorio. Los resultados evidenciaron diversas inconsistencias en los diferentes niveles de análisis, si bien la tendencia principal apunta a la confirmación de la estructura teórica. Teniendo en cuenta la validez predictiva mostrada por el TCI-R, se hace necesario un refinamiento que reafirme su valor clínico. Quizá, como sugiere el propio Cloninger, sea necesario utilizar metodologías no-lineales, que capten las relaciones dinámicas entre los sustratos neurológicos que subyacen a los rasgos y un concepto menos estático de los componentes de la personalidad.

Palabras clave: Personalidad; temperamento; carácter; adicción; análisis
\end{abstract} factorial multivariado; validez de constructo; TCI-R; Cloninger.

\section{Introducción}

Cloninger $(1986,1987)$ propuso su modelo biosocial de la personalidad, sustentado en la distinción entre rasgos temperamentales (basados en mecanismos biológicos) y caracteriales (producto del aprendizaje psicosocial), componentes ambos de la personalidad, tanto normal como patológica. De acuerdo con este modelo, el temperamento se refiere a respuestas emocionales automáticas que son heredables en una buena parte, se manifiestan desde los momentos iniciales de la vida y son estables en el tiempo. Por el contrario, los rasgos caracteriales representan diferencias individuales en autoconcepto, metas individuales y valores asumidos, que se desarrollan durante la vida a través de la experiencia compartida con el resto de personas. En su última formulación (Cloninger, Svrakic y Przybeck, 1993), se propusieron siete factores como componentes de la personalidad: búsqueda de novedad [NS], evitación del daño [HA], dependencia de recompensa [RD] y persistencia [PE], como rasgos temperamentales; y autodirección [SD], cooperación [CO] y autotrascendencia [ST], como rasgos caracteriales. Este modelo de la personalidad está racionalmente basado en conclusiones sólidas sobre las influencias neurobiológicas y de la experiencia en la estructura de la personalidad y el desarrollo (Cloninger, 2003; Cloninger, Svrakic y Svrakic, 1997).

Diversos estudios han explorado la evidencia empírica sobre la relación entre el sustrato biológico propuesto y cada

* Dirección para correspondencia [Correspondence address]: Eduardo J. Pedrero Pérez. CAD 4. Instituto de Adicciones. Ayuntamiento de Madrid. C/ Alcalá 527. 28027. Madrid (España).

E-mail: ejpedrero@yahoo.es
Title: Reliability and factorial validity of the TCI-R in a sample of addicts in treatment

Abstract: The TCI-R is the latest instrument to measure Cloninger biosocial theory and it has proven to be one of the most useful questionnaires in predicting behavior, especially in its pathological variants. Although the Spanish version was published seven years ago, there are few studies that support their psychometric goodness. It requires knowledge of the reliability and internal validity for clinical application. A sample of 824 subjects who started treatment for his addiction in a specialized center was recruited. Tests were performed to explore internal consistency of items, facets and main dimensions of the questionnaire, and construct validity studies using multivariate exploratory, parallel and confirmatory factor analysis. The results showed several inconsistencies in the different levels of analysis, although the main trend points to the confirmation of the theoretical structure. Given the predictive validity shown by the TCI-R, a refinement is necessary to reaffirm its clinical value. Perhaps, as suggested by Cloninger own, it will be necessary to use nonlinear methods, which capture the dynamic relationship between the neurological substrates underlying features and a less static concept of the components of personality.

Key words: Personality; temperament; character; addiction; multivariate factor analysis; construct validity; TCI-R; Cloninger.

una de las dimensiones temperamentales, lo que ha alcanzado cierto éxito en el caso de la NS. Aunque los primeros estudios se centraron en unidades genéticas específicas (Benjamín, Li, Patterson, Greenberg, Murphy y Hamer, 1996; Ebstein et al., 1996; Noble et al., 1998), los resultados de metaanálisis parecen abogar por mecanismos poligénicos (Schinka, Letsch y Crawford, 2002). Otros autores también encuentran una importante contribución genética a aquellos rasgos supuestamente independientes de la heredabilidad, como es el caso de la ST, relacionada con el córtex cerebral y con idéntica contribución dopaminérgica que la atribuida a la NS (Comings et al., 2000). En estudios electroencefalográficos también se ha encontrado evidencia de relación entre potenciales evocados y puntuaciones en el TCI, como la hallada entre el P300 y la dimensión de HA (Hansenne et al., 2003). Finalmente, estudios endocrinológicos dan soporte a la relación entre NS y neurotransmisión dopaminérgica central, pero no a la relación, propuesta por el modelo, entre RD y transmisión noradrenérgica (Hansenne et al., 2002).

Cloninger desarrolló, en primer lugar, un instrumento de medida, el TPQ (Cloninger, 1986; Cloninger, Przybeck y Svrakic, 1991), centrado en el estudio de las dimensiones temperamentales. La adición de dimensiones más ligadas al aprendizaje y la experiencia supuso el diseño de un nuevo instrumento, el TCI (Cloninger, Przybeck, Svrakic y Wetzel, 1994). Diversos problemas metodológicos -inconsistencia de la escala de PE, localización factorial de algunas subescalas de NS, RD y SD, poca variabilidad debida a la estructura de dos opciones de respuesta (Gutiérrez et al., 2001; Sato et al., 2001)- aconsejaron una nueva revisión y originaron el TCI-R (Cloninger, 1999). La versión revisada, el TCI-R, ha sido estudiada en varias lenguas, como francés (Hansenne, Delhez 
y Cloninger, 2005; Pelisolo et al., 2005), italiano (Fossati et al, 2007; Martinotti et al., 2008) y checo (Preiss, Kuchařová, Novák y Štěpánková, 2007).

Un estudio ha comparado la capacidad predictiva sobre la conducta de 11 cuestionarios de personalidad, encontrando que el NEO-PI-R y el TCI-R son los mejores predictores del comportamiento, siendo superior el TCI-R en la predicción de trastornos psicopatológicos (Grucza y Goldberg, 2007). Sin embargo, este estudio no agota el arsenal de instrumentos de medida actualmente disponibles, algunos de los cuales también han mostrado sobrada capacidad predictiva sobre variables psicopatológicas (p.e., el SNAP y el DAPP-BQ; Pryor, Miller y Gaughan, 2009).

A pesar de que el cuestionario está disponible desde hace 8 años, y que se ha utilizado en diversas poblaciones, apenas contamos con estudios que exploren la validez interna del TCI-R en su versión española. En español contamos con un estudio preliminar realizado sobre una muestra incidental de 400 personas de población general, de la que se obtuvieron datos normativos provisionales (Gutiérrez-Zotes et al., 2004), asumiendo la composición propuesta por los autores en la versión original en inglés, sin abordar el estudio de la estructura de la versión española. Recientemente se ha publicado un trabajo (Aluja, Blanch, Gallart y Dolcet, 2010) que explora la estructura factorial de la versión española y su relación con la edad en población no clínica; el análisis se efectúa al nivel de las subescalas del TCI-R, asumiendo la composición de ítems propuesta por los autores para cada una de ellas, siendo los resultados congruentes con los obtenidos en versiones en otros idiomas. No obstante, el TCI-R se ha utilizado, asumiendo la estructura teórica, para estimar diferencias de personalidad entre poblaciones clínicas con trastornos de la alimentación (Álvarez-Moya et al., 2007; Fernández-Aranda et al., 2006), psicosis (Cortés et al., 2009; Cortés Ruiz et al, 2010), trastornos adictivos (Pedrero Pérez, 2006a, 2007) y cáncer (Rodríguez et al., 2007), entre otros.

No se han encontrado trabajos que exploren la estructura del cuestionario TCI-R aplicado a muestras clínicas de adictos. Por otra parte, todos los trabajos previamente citados exploran la estructura a nivel de las subescalas, sin que se haya podido encontrar ningún análisis a nivel del ítem. Por ello, el objetivo principal del presente trabajo es conocer algunas propiedades psicométricas de la versión española del TCI-R en una población clínica (adictos a sustancias). En concreto, se pretende estudiar la consistencia interna de las escalas y subescalas, la estabilidad temporal, la tendencia de respuesta a los ítems y la estructura factorial del cuestionario, a nivel del ítem y al nivel de las subescalas.

\section{Método}

\section{Participantes}

Los participantes fueron reclutados por muestreo consecutivo de entre quienes iniciaban tratamiento por problemas derivados del uso de drogas en un centro especializado, público y gratuito (CAD-4 San Blas, Instituto de Adicciones. Ayuntamiento de Madrid) entre enero de 2007 y diciembre de 2010. La muestra final estuvo compuesta por 824 sujetos (191 mujeres) con una media de edad de 35.82 años (DT = 9.36). De ellos, 147 (36 mujeres) tenían como droga principal la heroína (edad $\mathrm{M}=37.24 ; D T=6.58), 332$ (66 mujeres) la cocaína $(M=32.00 ; D T=7,91), 292$ (81 mujeres) el alcohol $(M=41.45 ; D T=8.82)$ y 53 (8 mujeres) el cannabis $(M=26.66 ; D T=8.52)$. Sólo una pequeña parte de la muestra $(13.8 \%)$ contaba con estudios universitarios, el $28.9 \%$ estudios secundarios posobligatorios, el $43.0 \%$ estudios secundarios obligatorios y el $13.8 \%$ sólo estudios primarios.

\section{Instrumentos}

El Inventario de Temperamento y Carácter Revisado (Temperament and Character Inventory-Revised, TCI-R; Cloninger, 1999) es el instrumento más reciente para la evaluación de la personalidad derivado de la teoría biosocial de Cloninger. Se utilizó la traducción española del TCI-R, proporcionada directamente por los autores (Fernández-Aranda, Badía Casanovas, Bayón Pérez y Aitken, s/f; y resultados publicados en Gutiérrez-Zotes et al., 2004). El cuestionario consta de 240 ítems, de los cuales 5 son preguntas de validez (se pide que el participante marque una respuesta concreta). El resto miden búsqueda de novedad (NS; 35 ítems/4 subescalas), evitación del daño (HA; 33/4), dependencia de recompensa (RD; 30/4), persistencia (PE; 35/4), autodirección (SD; 40/5), cooperación (CO; 36/5) y autotrascendencia (ST; 26/3). Las respuestas se producen en una escala con formato de respuesta tipo Likert de 5 opciones (desde 'Falso' hasta 'Verdadero') y las puntuaciones se obtienen según el método de cálculo propuesto por Cloninger, mediante la suma del valor correspondiente a cada ítem (1-5, directamente o tras la inversión en los ítems formulados en negativo). Para explorar la consistencia temporal del cuestionario, éste se administró a una submuestra de 30 pacientes en dos ocasiones con un intervalo de presentación de 4 semanas.

\section{Procedimiento}

Los participantes cumplimentaron la prueba en el curso de una sesión clínica. Para su inclusión, todos los participantes debían cumplir criterios DSM-IV-TR de abuso/dependencia de al menos una sustancia en el momento de la evaluación, que se realizó entre la $2^{\mathrm{a}}$ y $4^{\mathrm{a}}$ semanas tras su entrada, una vez que se encontraban abstinentes a drogas no prescritas (lo que se constató mediante análisis toxicológicos de orina y de aire espirado). Como criterios de exclusión se establecieron: estado psicótico agudo $(n=2)$, retraso mental $(n=1)$, capacidad intelectual insuficiente $(n=6) \mathrm{y}$ conocimiento insuficiente del idioma español $(n=5)$. Se excluyeron también los cuestionarios incompletos, mal cumplimentados o que puntuaban 2 o más en la escala de validez $(n=24)$. Todos ellos fueron informados del doble propósito de la evaluación: clínico, para orientar el psicodiagnóstico y 
el programa individualizado de tratamiento, y de investigación científica. También recibieron información sobre sus derechos y se solicitó su consentimiento informado para el uso anónimo de sus datos.

\section{Análisis de los datos}

Se realizaron pruebas de fiabilidad para las escalas y los ítems, así como un análisis de tendencias de respuesta a los ítems. Puesto que la modalidad de respuesta del TCI-R es una escala Likert de 5 puntos, los análisis univariados corren el riesgo de agrupar a los ítems según su distribución y no según su contenido, proporcionando resultados espurios (Bernstein y Teng, 1989; Muthén y Kaplan, 1985, 1992). Por ello, mediante el programa FACTOR 8.02 (Lorenzo-Seva y Ferrando, 2006), se efectuó un análisis factorial exploratorio multivariado; se obtuvo, en primer lugar, la matriz de correlaciones policóricas, utilizando el criterio de Mardia (1970) para garantizar la normalidad multivariada. A continuación se utilizaron dos métodos para determinar el número de factores a retener: un análisis paralelo optimizado (Timmerman y Lorenzo-Seva, 2011) y una prueba de mínima correlación parcial promediada (MAP; Velicer, 1976). Seguidamente se efectuó un análisis factorial de rangos mínimos (Ten Berge y Kiers, 1991) y una rotación Oblimin directo. Entre las subescalas se realizaron pruebas de correlación de orden cero y parciales, controlando covariables y se efectuaron análisis exploratorios con rotación Promax. Estos análisis se realizaron con el paquete estadístico SPSS 15.0 (SPSS Inc., Chicago, IL). Se efectuó un análisis factorial confirmatorio utilizando el programa AMOS 6.0 (Arbuckle, 2005).

\section{Resultados}

\section{Consistencia interna}

En la Tabla 1 se detallan los estadísticos de consistencia interna de las escalas, subescalas e ítems del TCI-R. Todas las escalas mostraron valores $a$ de Cronbach satisfactorios. En promedio, la consistencia interna de las subescalas resultó también aceptable (mediana $a=.72$, percentil $25=.64$, percentil $75=.77$ ), con la excepción de 11 de ellas, con un $a$ $<$.70. Los valores de correlación total corregida de los ítems resultaron también en el rango entre aceptables y buenos; sin embargo, en varias escalas (principalmente NS, RD y CO) una minoría no despreciable de los elementos no mostró una consistencia satisfactoria $(r<.20)$.
Tabla 1. Estadísticos descriptivos de consistencia interna de las escalas y subescalas del TCI - R.

\begin{tabular}{|c|c|c|c|c|c|c|}
\hline \multirow[b]{3}{*}{ CI-R Escalas y subescalas } & \multirow{3}{*}{$r_{i j}^{-}$} & \multirow{3}{*}{$n$} & \multicolumn{4}{|c|}{$\%$ ítems $\%$ ítems } \\
\hline & & & $\alpha$ & con & $\operatorname{con}$ & $r^{-}$ \\
\hline & & & & $r_{i t}<.20$ & $r_{i t}<.10$ & \\
\hline Excitabilidad Exploratoria (NS1) & .10 & 10 & .54 & 30.0 & 20.0 & .23 \\
\hline Impulsividad (NS2) & .20 & 9 & .72 & 11.1 & 0.0 & .39 \\
\hline Extravagancia (NS3) & .23 & 9 & .72 & 33.3 & 11.1 & .41 \\
\hline Desorden (NS4) & .14 & 7 & .53 & 42.9 & 0.0 & .26 \\
\hline Búsqueda de Novedad (total) & .09 & 35 & .79 & 31.4 & 8.6 & .27 \\
\hline Preocupación anticipatoria (HA1) & .19 & 11 & .72 & 9.1 & 0.0 & .37 \\
\hline Miedo a la incertidumbre (HA2) & .21 & 7 & .66 & 0.0 & 0.0 & .37 \\
\hline Timidez (HA3) & .32 & 7 & .77 & 0.0 & 0.0 & .49 \\
\hline Fatigabilidad (HA4) & .26 & 8 & .74 & 0.0 & 0.0 & .43 \\
\hline Evitación del Daño (total) & .17 & 33 & .86 & 3.0 & 0.0 & .37 \\
\hline Sentimentalismo (RD1) & .16 & 8 & .61 & 0.0 & 0.0 & .31 \\
\hline Cordialidad (RD2) & .26 & 10 & .78 & 0.0 & 0.0 & .44 \\
\hline Apego (RD3) & .37 & 6 & .78 & 0.0 & 0.0 & .53 \\
\hline Dependencia (RD4) & .16 & 6 & .54 & 33.3 & 0.0 & .28 \\
\hline Dependencia de Recompensa (total) & .14 & 30 & .88 & 23.3 & 6.7 & .35 \\
\hline Capacidad de esfuerzo (P1) & .21 & 9 & .69 & 11.1 & 0.0 & .37 \\
\hline Trabaj & .24 & 8 & .71 & 0.0 & 0.0 & .40 \\
\hline Ambición (P3) & .25 & 10 & .77 & 0.0 & 0.0 & .44 \\
\hline Perfeccionismo (P4) & .22 & 8 & .69 & 12.5 & 0.0 & .39 \\
\hline Persistencia (total) & .19 & 35 & .89 & 2.9 & 0.0 & .41 \\
\hline Responsabilidad (SD1) & .31 & 8 & .79 & 0.0 & 0.0 & .49 \\
\hline Orientación (SD2) & .31 & 6 & .73 & 0.0 & 0.0 & .47 \\
\hline s (SD3) & .28 & 5 & .67 & 0.0 & 0.0 & .42 \\
\hline ptación (SD4) & .27 & 10 & .78 & 0.0 & 0.0 & .45 \\
\hline Congruencia (SD5) & .19 & 11 & .72 & 9.1 & 0.0 & .36 \\
\hline Autodirección (total) & .16 & 40 & .89 & 7.5 & 2.5 & .37 \\
\hline Tolerancia (CO1) & .27 & 8 & .74 & 0.0 & 0.0 & .44 \\
\hline Empatía (CO2) & .21 & 5 & .56 & 20.0 & 0.0 & .33 \\
\hline Altruismo (CO3) & .12 & 8 & .48 & 37.5 & 12.5 & .23 \\
\hline Compasión (CO4) & .48 & 7 & .89 & 0.0 & 0.0 & .64 \\
\hline Integridad (CO5) & .10 & 8 & .46 & 37.5 & 12.5 & .20 \\
\hline Cooperación (total) & .15 & 36 & .86 & 13.9 & 2.8 & .36 \\
\hline Ensimismamiento (ST1) & .19 & 10 & .70 & 0.0 & 0.0 & .36 \\
\hline Transpersonalidad (ST2) & .25 & 8 & .72 & 0.0 & 0.0 & .42 \\
\hline Espiritualidad (ST3) & .31 & 8 & .78 & 0.0 & 0.0 & .48 \\
\hline Autotrascendencia (total) & .18 & 26 & .85 & 7.7 & 0.0 & .40 \\
\hline
\end{tabular}

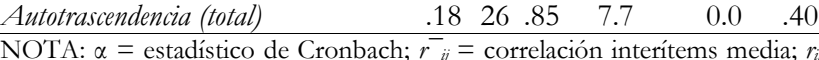
$=$ correlación ítem-total corregida; $r{ }^{-}$it $=$correlación ítem-total corregida media; $n=$ número de ítems.

\section{Análisis de tendencias de respuesta}

La media de porcentajes observados para cada categoría de respuestas en la escala de 5 puntos fue la siguiente:

- respuesta 1 (falso): $17.93 \%$ [ítems directos 18.26\%; ítems inversos $17.44 \%$ ].

respuesta 2 (probablemente falso): $15.10 \%$ [ítems directos 12.62 ; ítems inversos $18.02 \%$ ].

respuesta 3 (ni cierto ni falso, igual cierto que falso): $20.60 \%$ [ítems directos $20.92 \%$; ́items inversos $20.24 \%$ ]

respuesta 4 (probablemente verdadero): $20.23 \%$ [ítems directos $23.37 \%$; ítems inversos $16.66 \%$ ].

respuesta 5 (verdadero): $26.16 \%$. [ítems directos $24.84 \%$; ítems inversos $27.64 \%$ ] 
Atendiendo a las tendencias de respuesta, resultaba de interés conocer si existe una preferencia por las puntuaciones extremas (1. Falso y 5. Verdadero), lo que denotaría un pensamiento de tipo dicotómico, frecuentemente asociado a diversas formas de psicopatología. La media de la suma de elecciones extremas por sujeto fue de 48.95 ítems $(D T=$ 23.41 ), lo que supone un $18.70 \%$ de las respuestas en cada cuestionario. Un $2.43 \%$ de los sujetos alcanzaron un número de ítems extremos correspondientes a la media más 2 desviaciones típicas. Por el contrario, y puesto que el TCI-R ofrece la posibilidad de responder "ni cierto ni falso, igual cierto que falso" (respuesta 3), resultaba de interés saber si algunos ítems recibían con más frecuencia esta respuesta elusiva. Los cinco ítems con mayor frecuencia de respuesta neutral fueron el 84 de CO3 (51.6\%), el 232 de ST3 (41.2\%), el 76 de PS4 (37.7\%), el 220 de SD5 (34.6\%) y el 203 de CO1 (34.3\%). Ninguno de estos ítems coincide con los encontrados en el trabajo de Pelissolo et al. (2005), pero, como en este trabajo, nuestros datos también encuentran una ligera, pero significativa correlación entre el porcentaje de respuestas ' 3 ' y la numeración del ítem $(r=.18$; $p<.001)$.

\section{Estabilidad test-retest}

Con objeto de estudiar la estabilidad temporal, el TCI-R fue administrado en dos ocasiones, tras un intervalo de 4 semanas, a un grupo de 30 pacientes (15 varones y 15 mujeres, edad media 34.6 años; $D T=11.4)$. Las puntuaciones medias de las siete escalas principales del TCI-R y los coeficientes de correlación intraclase (CCI) se presentan en la Tabla 2. Todos los CCIs se situaron por encima de .80, con la excepción de la CO.

Tabla 2. Resultados del estudio test-retest para las principales dimensiones del TCI-R $(n=30)$.

\begin{tabular}{llllc}
\hline & T1 & T2 & $C C I$ & $p$ \\
\hline Búsqueda de novedad & $115.3 \pm 13.2$ & $112.6 \pm 10.5$ & .85 & $<.0001$ \\
Evitación del daño & $106.2 \pm 20.2$ & $106.8 \pm 15.4$ & .93 & $<.0001$ \\
$\begin{array}{l}\text { Dependencia de } \\
\quad \text { recompensa }\end{array}$ & $97.4 \pm 19.1$ & $101.0 \pm 15.3$ & .95 & $<.0001$ \\
Persistencia & $99.8 \pm 17.1$ & $99.5 \pm 16.7$ & .95 & $<.0001$ \\
Autodirección & $120.8 \pm 22.7$ & $132.1 \pm 19.4$ & .88 & $<.0001$ \\
Cooperación & $130.6 \pm 16.9$ & $135.5 \pm 13.4$ & .76 & $<.0001$ \\
Autotrascendencia & $70.3 \pm 15.1$ & $70.8 \pm 14.4$ & .87 & $<.0001$ \\
\hline $\begin{array}{l}\text { Nota: } \text { T1 = administración del TCI-R en tiempo 1; T2 } \\
\text { eficiente de correlación intraclase. }\end{array}$ & & & &
\end{tabular}

\section{Análisis factorial exploratorio multivariado a nivel del ítem}

Se configuró, en un primer paso, la matriz de correlaciones policóricas, que alcanzó el criterio de normalidad multivariada (Asimetría 20508.16; $g l=2190670$; Curtosis 60500.43; $g l=206.7$; estadístico de Mardia 206.65; $p<$ .0001). Esta matriz de correlaciones policóricas mostró adecuados indicadores de adecuación para el procedimiento factorial (estadístico de Barlett $=88861.3 ; g l=27495 ; p<$
.0001 ; Kaiser-Meyer-Olkin KMO $=.869$; $p<.0001)$. A continuación, y sobre esta matriz, se efectuó un análisis paralelo optimizado (con extracción aleatoria de 500 submatrices), basado en un análisis de rangos mínimos, que proporcionó una solución de 16 factores (Tabla 3), que explicaban el $44,6 \%$ de la varianza total. Por el contrario, la prueba de mínima correlación parcial promediada $(M A P)$ ofreció una solución de 7 factores, si bien sólo podía explicar un 33.5\% de la varianza compartida.

Tabla 3. Análisis paralelo y prueba de mínima correlación parcial promediada $(M A P)($ items $=235 ; N=824)$.

\begin{tabular}{|c|c|c|c|c|}
\hline & \multicolumn{3}{|c|}{$\begin{array}{c}\text { Autovalores actuales y aleatoriamente } \\
\text { obtenidos }\end{array}$} & $M A P$ \\
\hline Factor & $\begin{array}{c}\text { Autovalor } \\
\text { Normal }\end{array}$ & $\begin{array}{c}\text { Autovalor } \\
\text { Medio }\end{array}$ & Percentil 95 & $\begin{array}{c}\text { Promedio } \\
\text { parcial }\end{array}$ \\
\hline 1 & 21.314 & 2.346 & 2.398 & .0102 \\
\hline 2 & 16.056 & 2.295 & 2.335 & .0045 \\
\hline 3 & 9.704 & 2.258 & 2.293 & .0031 \\
\hline 4 & 7.568 & 2.224 & 2.257 & .0025 \\
\hline 5 & 4.753 & 2.196 & 2.226 & .0023 \\
\hline 6 & 4.350 & 2.171 & 2.198 & .0023 \\
\hline 7 & 3.929 & 2.145 & 2.171 & .0022 \\
\hline 8 & 3.458 & 2.122 & 2.146 & .0024 \\
\hline 9 & 3.067 & 2.100 & 2.123 & .0026 \\
\hline 10 & 2.872 & 2.078 & 2.102 & .0028 \\
\hline 11 & 2.730 & 2.058 & 2.079 & \\
\hline 12 & 2.642 & 2.038 & 2.059 & \\
\hline 13 & 2.306 & 2.019 & 2.040 & \\
\hline 14 & 2.169 & 2.000 & 2.020 & \\
\hline 15 & 2.138 & 1.983 & 2.003 & \\
\hline 16 & 2.075 & 1.965 & 1.983 & \\
\hline 17 & 1.924 & 1.948 & 1.966 & \\
\hline 18 & 1.856 & 1.931 & 1.948 & \\
\hline 19 & 1.832 & 1.914 & 1.931 & \\
\hline 20 & 1.780 & 1.898 & 1.916 & \\
\hline
\end{tabular}

Se trabajó sobre esta solución de 7 factores por ser la que se asemejaba a la estructura teóricamente propuesta. Sobre la matriz de correlaciones policóricas entre los 235 ítems se forzó la solución de 7 factores y se efectuó una rotación oblicua (Oblimin directo). El primer factor agrupó, casi con total exactitud, a los ítems de ST, con cargas factoriales entre .75 y .14 , aunque el $15.4 \%$ de los ítems de esta escala presentaba su máxima carga en algún otro factor. En el segundo factor fueron la mayor parte de los ítems de SD los que presentaron la carga principal $(60.0 \%)$, si bien el factor en su conjunto presentaba una mescolanza de ítems de toda procedencia y un carácter bipolar, siendo el polo positivo el más relacionado con la SD y el negativo compuesto casi en exclusiva por ítems procedentes de HA y RD. El tercer factor agrupaba casi con total exactitud a los ítems de PE, aunque sólo el 80\% de los ítems de esta escala presentaba su carga principal en este factor, mientras que un $14.3 \%$ cargaban principalmente en el polo positivo del segundo factor. El cuarto factor agrupaba principalmente a ítems de HA, aunque sólo un $60.6 \%$ de ellos presentaba en él su carga principal, en tanto que un $36.4 \%$ lo hacía en el polo negativo del 
segundo factor. El quinto factor agrupó mayoritariamente a ítems de NS, aunque sólo un $57.1 \%$ presentaban su carga principal en este factor, en tanto que un $25.7 \%$ lo hacían en el polo positivo del segundo factor (lo cual era más frecuente en la subescala de NS1 (60\%). El sexto factor agrupaba prioritariamente y con cargas máximas a los ítems de RD, aunque sólo un 56.8 de ellos presentaba su carga principal en este factor y un $20.0 \%$ lo hacía en el polo negativo del segundo factor. Por último, el séptimo factor agrupaba principalmente a ítems de CO, siendo el $61.1 \%$ de los ítems de esta escala los que presentaban su carga máxima en él, en tanto que un $13.9 \%$ lo hacían en el polo positivo del segundo factor.

\section{Análisis a nivel de escalas y subescalas}

La Tabla 4 muestra la matriz de intercorrelaciones de las cuatro escalas de temperamento y las tres de carácter, mediante correlación de orden 0 o controlando la edad como covariable (ya que mostró correlaciones de signo diverso con algunas dimensiones del TCI-R). Si observamos sólo las escalas temperamentales se puede apreciar que la HA mostró correlaciones negativas y significativas con las otras tres escalas (con la NS únicamente cuando se controla la edad), con mayor tamaño del efecto en el caso de la PE. En cuanto a las caracteriales, la correlación entre SD y CO presentó un considerable tamaño del efecto. Tomadas todas en conjunto, destacan las correlaciones negativas entre SD, por un lado, $y$ NS y HA por otro, en tanto que la CO y la RD correlacionan entre sí con considerable tamaño del efecto.

Tabla 4. Matriz de intercorrelaciones de las escalas principales de temperamento y carácter del TCI-R $(N=824)$.

\begin{tabular}{|c|c|c|c|c|c|c|c|}
\hline & NS & HA & RD & PE & SD & $\mathrm{CO}$ & Edad \\
\hline NS & & & & & & & $-.26 *$ \\
\hline $\mathrm{HA}$ & $\begin{array}{c}-.18^{*} \\
\left(.14^{*}\right)\end{array}$ & & & & & & $.19 *$ \\
\hline $\mathrm{RD}$ & $\begin{array}{l}.05 \\
(.04)\end{array}$ & $\begin{array}{c}-.20 * \\
\left(-.19^{*}\right)\end{array}$ & & & & & -.05 \\
\hline PE & $\begin{array}{l}-.05 \\
(.08)\end{array}$ & $\begin{array}{c}-.42^{*} \\
\left(-.41^{*}\right)\end{array}$ & $\begin{array}{c}.19^{*} \\
\left(.18^{*}\right)\end{array}$ & & & & -.10 \\
\hline SD & $\begin{array}{c}-.37^{*} \\
\left(-.37^{*}\right)\end{array}$ & $\begin{array}{c}-.50 * \\
\left(-.52^{*}\right)\end{array}$ & $\begin{array}{c}.19^{*} \\
\left(.19^{*}\right)\end{array}$ & $\begin{array}{c}.27^{*} \\
\left(.28^{*}\right)\end{array}$ & & & .05 \\
\hline $\mathrm{CO}$ & $\begin{array}{c}-.25^{*} \\
\left(-.23^{*}\right)\end{array}$ & $\begin{array}{c}-.21 * \\
\left(-.24{ }^{*}\right)\end{array}$ & $\begin{array}{c}.54^{*} \\
\left(.54^{*}\right)\end{array}$ & $\begin{array}{c}.15^{*} \\
\left(.17^{*}\right)\end{array}$ & $\begin{array}{c}.52 * \\
(.52 *)\end{array}$ & & .11 \\
\hline ST & $\begin{array}{c}.09 \\
(.12)\end{array}$ & $\begin{array}{l}-.03 \\
(-.05)\end{array}$ & $\begin{array}{c}.23^{*} \\
\left(.24^{*}\right)\end{array}$ & $\begin{array}{c}.34^{*} \\
\left(.35^{*}\right)\end{array}$ & $\begin{array}{c}-.15^{*} \\
\left(-.16^{*}\right)\end{array}$ & $\begin{array}{c}.11 \\
(.11)\end{array}$ & .09 \\
\hline
\end{tabular}

Nota: Entre paréntesis correlaciones parciales controlando la edad. $*=\mathrm{Co}-$ rrelaciones significativas tras aplicación de la corrección de Bonferroni para comparaciones múltiples $(p<.001)$. En negrita, correlaciones con mayor tamaño del efecto.

A continuación, se realizó un análisis exploratorio con la extracción de ejes principales, rotación oblicua Promax (kap$p a=4)$ para el conjunto de las 29 subescalas del TCI-R (Tabla 5). Se identificaron siete factores, que explican $64.96 \%$ de la varianza total.
Tabla 5. Análisis factorial exploratorio $(N=824)$ tomando las 29 subescalas del TCI-R; 7 factores con autovalor $\geq 1$ explicando el $64.96 \%$ de la varianza total.

\begin{tabular}{|c|c|c|c|c|c|c|c|}
\hline & \multicolumn{7}{|c|}{ Factores } \\
\hline & $\begin{array}{c}1 \\
(\mathrm{SD} / \mathrm{HA})\end{array}$ & $\begin{array}{c}2 \\
(\mathrm{PE})\end{array}$ & $\begin{array}{c}3 \\
(\mathrm{NS})\end{array}$ & $\begin{array}{c}4 \\
(\mathrm{RD})\end{array}$ & $\begin{array}{c}5 \\
(\mathrm{C})\end{array}$ & $\begin{array}{c}6 \\
(\mathrm{ST})\end{array}$ & $\begin{array}{c}7 \\
\text { ('odd man out') }\end{array}$ \\
\hline NS1 & .45 & .29 & -.27 & .46 & -.20 & .33 & .32 \\
\hline NS2 & -.18 & -.18 & -.76 & -.12 & -.24 & -.02 & -.34 \\
\hline NS3 & -.11 & -.02 & -.71 & .11 & -.14 & .12 & -.08 \\
\hline NS4 & .06 & -.15 & -.65 & .06 & -.40 & .08 & -.36 \\
\hline HA1 & -.76 & -.21 & -.14 & -.19 & -.15 & .03 & .00 \\
\hline HA2 & -.63 & -.22 & .24 & -.24 & .19 & -.16 & .16 \\
\hline HA3 & -.52 & -.24 & .05 & -.65 & -.14 & -.14 & -.01 \\
\hline HA4 & -.73 & -.41 & -.16 & -.26 & -.09 & .01 & -.16 \\
\hline RD1 & -.33 & .32 & -.19 & .41 & .44 & .40 & .12 \\
\hline RD2 & .22 & .13 & .13 & .80 & .16 & .13 & .38 \\
\hline RD3 & .21 & .34 & -.05 & .90 & .28 & .26 & .16 \\
\hline RD4 & -.03 & -.05 & .25 & .13 & .33 & -.20 & .68 \\
\hline PE1 & .21 & .79 & -.04 & .25 & .12 & .23 & .01 \\
\hline PE2 & .45 & .80 & .31 & .30 & .20 & .28 & .08 \\
\hline PE3 & .32 & .75 & .01 & .33 & -.30 & .34 & -.10 \\
\hline PE4 & .30 & .83 & .31 & .22 & .09 & .31 & .15 \\
\hline SD1 & .65 & -.03 & .44 & .15 & .24 & -.30 & .42 \\
\hline SD2 & .61 & .46 & .51 & .32 & .13 & .07 & .28 \\
\hline SD3 & .75 & .46 & .36 & .32 & .15 & .02 & .30 \\
\hline SD4 & .21 & -.24 & .44 & -.17 & .55 & -.33 & .40 \\
\hline SD5 & .59 & .32 & .64 & .27 & .33 & -.05 & .27 \\
\hline CO1 & .30 & .13 & .31 & .35 & .72 & -.04 & .31 \\
\hline $\mathrm{CO} 2$ & .19 & .36 & .21 & .52 & .55 & .27 & .22 \\
\hline $\mathrm{CO} 3$ & .22 & .27 & .29 & .43 & .55 & .03 & .61 \\
\hline $\mathrm{CO} 4$ & .09 & .09 & .24 & .17 & .77 & .05 & .39 \\
\hline $\mathrm{CO} 5$ & .08 & .07 & .24 & .16 & .37 & .12 & .77 \\
\hline ST1 & -.13 & .45 & -.29 & .21 & -.02 & .80 & -.29 \\
\hline ST2 & .00 & .40 & .05 & .35 & .17 & .82 & .01 \\
\hline ST3 & -.09 & .17 & -.05 & .14 & .01 & .76 & .11 \\
\hline
\end{tabular}

Nota. Extracción de componentes principales, con rotación oblicua Promax (kappa=4) y normalización con Kaiser.

La supuesta estructura de siete factores ofrece varias inconsistencias. El séptimo factor no se puede asignar a ninguna de las dimensiones principales, recogiendo ciertas cargas superiores a las que algunas subescalas muestran en el factor en el que sería esperable que lo hicieran, principalmente CO5 "Integridad" (.77), RD4 "Dependencia" (.68) y CO3 "Altruismo" (.61). Este séptimo factor parece representar una "categoría de elementos raros" ("odd man out category"; Unser, Steven y Trus, 1986), aquellas dimensiones que no caben en los seis factores anteriores y tienden a formar una nueva. Las subdimensiones de HA y SD cargan en el mismo factor 1, con signo contrario; aunque no pueda afirmarse que se trate de un factor bipolar en toda su extensión, se ob- 
serva una tendencia notable derivada de esta doble ocupación.

Especial atención merecen las subescalas RD4 y NS1: NS1 muestra una incierta pertenencia factorial, cargando hasta en 4 factores por encima de lo que lo hace en el que debiera serle propio, al igual que RD4, y ambos cargan notablemente en ese 'extraño' séptimo factor.

Tabla 6. Análisis factorial exploratorio $(N=824)$ de las 16 subescalas temperamentales del TCI-R, 4 factores con autovalor $\geq 1$ explicando el $61.56 \%$ de la varianza total.

\begin{tabular}{|c|c|c|c|c|}
\hline & \multicolumn{4}{|c|}{ Factores } \\
\hline & $1(\mathrm{PE})$ & 2 (RD) & 3 (HA) & 4 (NS) \\
\hline NS1 & .31 & .49 & -.42 & .33 \\
\hline NS2 & -.19 & -.09 & .04 & .76 \\
\hline NS3 & -.02 & .18 & .08 & .69 \\
\hline NS4 & -.13 & -.02 & -.22 & .73 \\
\hline HA1 & -.26 & -.15 & .76 & .02 \\
\hline HA2 & -.27 & -.16 & .75 & -.35 \\
\hline HA3 & -.26 & -.61 & .56 & -.14 \\
\hline HA4 & -.44 & -.24 & .72 & .09 \\
\hline RD1 & .27 & .50 & .53 & .10 \\
\hline RD2 & .10 & .82 & -.13 & -.10 \\
\hline RD3 & .31 & .89 & -.16 & .08 \\
\hline RD4 & -.13 & .30 & .19 & -.40 \\
\hline PE1 & .80 & .23 & -.18 & .06 \\
\hline PE2 & .82 & .24 & -.38 & -.25 \\
\hline PE3 & .76 & .16 & -.35 & .10 \\
\hline PE4 & .85 & .18 & -.23 & -.28 \\
\hline
\end{tabular}

Nota: Extracción de componentes principales, con rotación oblicua Promax (kappa=4) y normalización con Kaiser. Cargas en las escalas principales en negrita.

A continuación, se realizó un análisis factorial exploratorio con extracción de componentes principales, rotación oblicua Promax (kappa $=4)$, tomando por separado las cuatro dimensiones temperamentales (Tabla 6) y las tres caracteriales (Tabla 7). Al explorar las dimensiones temperamentales aparecieron cuatro factores (varianza total explicada $61.6 \%$ ), si bien persistieron las inconsistencias previamente detectadas. Todas las subescalas de temperamento mostraron mayores saturaciones en sus cuatro dimensiones teóricas principales (Tabla 6), a excepción nuevamente de NS1, RD1 y RD4, que presentan cargas superiores y de distinto signo en factores diferentes al teóricamente asignado.

En cuanto a las dimensiones caracteriales por separado (Tabla 7), tras la extracción de componentes principales se identificaron tres factores con valores propios superiores a 1 , explicando $59.34 \%$ de la varianza total. Tras la rotación oblicua Promax $(k a p p a=4)$, todas las escalas saturan en consonancia con la propuesta teórica, a excepción de SD4 "Autoaceptación".
Tabla 7. Análisis factorial exploratorio $(N=824)$ de las 13 subescalas caracteriales del TCI-R, 4 factores con autovalor $\geq 1$ explicando el $59.4 \%$ de la varianza total.

\begin{tabular}{lccc}
\hline & \multicolumn{3}{c}{ Factores } \\
\cline { 2 - 4 } & $1(\mathrm{C})$ & $2(\mathrm{SD})$ & $3(\mathrm{ST})$ \\
\hline SD1 & .44 &. $\mathbf{6 9}$ & -.36 \\
SD2 & .27 &. $\mathbf{8 2}$ & .09 \\
SD3 & .32 & .82 & .00 \\
SD4 & .59 & .27 & -.41 \\
SD5 & .43 & .79 & -.04 \\
CO1 & .71 & .47 & -.01 \\
CO2 & .55 & .44 & .35 \\
CO3 & .73 & .44 & .07 \\
CO4 & .76 & .24 & .05 \\
CO5 & .68 & .19 & .06 \\
ST1 & -.15 & -.08 & .83 \\
ST2 & .17 & .14 & .84 \\
ST3 & .09 & -.07 & .69
\end{tabular}

Nota: Extracción de componentes principales, con rotación oblicua Promax (kappa $=4)$ y normalización con Kaiser. Cargas en las escalas principales en negrita.

\section{Estructura factorial: análisis factorial confirmatorio}

Asumiendo un modelo teórico de cuatro factores para el análisis de las escalas temperamentales, los resultados muestran un pobre ajuste de los datos $\left(\chi^{2}=1258.62 ; g l=98 ; p\right.$ $<.001 ; \chi^{2} / d f=12.84 ;$ RMSEA $=.12$ [IC del $90 \%=.11-.13$ ]; $\mathrm{CFI}=.70 ; \mathrm{PGFI}=.60)$, no mejorando sustancialmente si se eliminan, en un primer paso, las subescalas NS1 y RD4, ni en un segundo paso, RD1. En cuanto a las dimensiones caracteriales, y asumiendo un modelo de tres factores, tampoco son admisibles los indicadores de ajuste, ni siquiera eliminando la subescala SD4 $\left(\chi^{2}=660.12 ; g l=62 ; p<.001 ; \chi^{2} / d f\right.$ $=10.65$; RMSEA = .11 [IC del $90 \%=.10-.12$ ]; CFI = .67; PGFI $=.60)$.

\section{Discusión}

El TCI-R debe considerarse, a tenor de la literatura disponible, uno de los instrumentos de mayor utilidad clínica para el estudio de la personalidad, tanto en su vertiente normal como en diversas condiciones clínicas. Sin embargo, aunque disponemos de diversos estudios sobre la estructura factorial de este instrumento, todos ellos parten de asumir la composición de ítems originalmente propuesta por los autores para las subescalas. El presente estudio es el primero que estudia la estructura factorial del TCI-R partiendo del nivel del ítem.

La mayor parte de los estudios factoriales sobre cuestionarios parten de la asunción errónea de que las escalas tipo Likert pueden considerarse como escalas continuas y, en consecuencia, aplican análisis lineales univariados a partir de la matriz de correlaciones de Pearson. Los procedimientos de análisis factorial más usuales (p. e., análisis factorial común, de máxima verosimilitud y de componentes principales) producen resultados significativos solamente si los datos utilizados son verdaderamente continuos y multivariadamente normales. Los datos a nivel del ítem, que frecuen- 
temente se usan en la investigación psicológica, habitualmente basados en escalas tipo Likert, casi nunca reúnen estos requerimientos, proporcionando resultados espurios, que agrupan los ítems más por la similitud de sus distribuciones que con base en sus contenidos (Bernstein y Teng, 1989). Por ello, en el presente trabajo se ha adoptado la estrategia de análisis basada en la matriz de correlaciones policóricas, en tanto que este tipo de correlación estima la relación lineal entre dos variables latentes continuas que subyacen a dos variables observadas ordinales que son indicadores manifiestos de aquellas (Flora y Curran, 2004). A partir de esta matriz se han utilizado estrategias para determinar el número de factores a retener y la estructura factorial rotada, a partir de las técnicas ofrecidas por el programa FACTOR (Lorenzo-Seva y Ferrando, 2006), gratuito y disponible en Internet.

La estructura factorial encontrada cuando se analizan los 235 ítems del TCI-R (una vez excluidos los 5 de validez) presenta una adecuación moderada a la propuesta teórica, pero también severas inconsistencias. En términos generales, cada uno de los factores hallados se asemeja a una de las escalas principales de la propuesta teórica. Sin embargo, los ítems que las componen sólo se asemejan a los teóricamente esperables entre un $55 \%$ y un $80 \%$. De especial interés es ese segundo factor que agrupa a buena parte de los ítems de SD, pero también a porcentajes significativos de otras escalas, en una distribución bipolar. Estos datos sugieren que la SD es una dimensión difícilmente diferenciable de otras teóricamente diversas. Más adelante se sugerirá una explicación a este hecho.

Cuando se estudió la estructura factorial del TCI-R en el nivel de las subescalas, ésta presentó importantes inconsistencias, especialmente en las dimensiones temperamentales. Los problemas principales observados se refieren a inconsistencia de algunos ítems (especialmente en las subescalas NS1, NS3, CO3 y CO5) y de algunas escalas (principalmente $\mathrm{NS}, \mathrm{RD}$ y CO), y una estructura factorial que muestra un precario ajuste entre los datos y la teoría en que se sustenta el cuestionario. Los resultados obtenidos en el presente estudio son muy similares a los obtenidos en población general española (Aluja et al., 2010), que también encontraron una pobre consistencia en las escalas NS1 $(\alpha=.44)$, CO3 ( $\alpha=$ .52) y $\operatorname{CO} 5(\alpha=.54)$, pero también replican en buena parte lo obtenido en otros entornos culturales, con otras poblaciones y en diversos idiomas: Pelissolo et al. (2005) informan también de problemas con NS1 en la versión francesa; Preiss et al. (2007) encontraron problemas con NS1 y RD4 en la versión checa; Martinotti et al. (2008) tuvieron también dificultades con RD4, entre otras subescalas dudosas en la versión italiana; Hansenne et al. (2005) encontraron graves problemas con NS1 en una muestra de Bélgica. En contraste con estos hallazgos Fossati et al. (2007), en una muestra de sujetos italianos, informa de haber encontrado una estructura lineal factorial que está muy cerca de la esperada.

Todos estos hallazgos en su conjunto parecen implicar que la mayoría de los problemas psicométricos que condujeron a la revisión del TCI aún permanecen en el TCI-R, con excepción de los problemas con la PE. En concordancia con lo observado por otros autores, los datos del presente estudio inciden en la necesidad de considerar cuestionable la pertenencia de NS1 (excitabilidad exploratoria) a la dimensión general de búsqueda de novedad: Aluja et al. (2010) encontraron que NS1 no carga en el factor de NS, presentando cargas positivas en RD y negativas en HA. Pedrero Pérez (2009a) encontró correlaciones negativas o inexistentes entre NS1 y el resto de subdimensiones de NS, tanto en adictos como en muestras no clínicas; Álvarez-Moya et al. (2010) encontraron resultados similares en jugadores patológicos; la subdimensión NS1 no predice trastornos de personalidad (Pedrero Pérez, 2006a) y, al contrario que el resto de subdimensiones de NS, no aparecen puntuaciones elevadas en poblaciones de adictos (Pedrero Pérez y Rojo Mota, 2008). Además, NS1 muestra correlaciones estables con la dimensión impulsividad funcional de Dickman, mientras que las otras subdimensiones de NS correlacionan positivamente con la dimensión de impulsividad disfuncional: la excitabilidad exploratoria (NS1) podría estar relacionada con dimensiones de personalidad como búsqueda de sensaciones de Zuckerman, $B A S$ de Gray o atrevimiento, desinhibición y alegria de Eysenck (venturesomeness, desinbibition y liveliness) (Pedrero Pérez, 2009a, b). Estos hallazgos conducen a la hipótesis de que los sustratos biológicos de la NS1 pueden ser diferentes de los de las restantes subdimensiones de NS: tal vez NS1 está más relacionada con la regulación de los comportamientos aproximativos, en busca de estimulación y de refuerzo (como implica su carga notable en factor de RD), la curiosidad y la exploración del ambiente, funciones que parecen estar relacionados con la actividad del núcleo estriado. Todas las restantes subdimensiones de NS pueden estar más relacionadas con diferencias en la actividad dopaminérgica de los circuitos corticofrontales. Esta es una de las posibles razones por las cuales, como se ha discutido anteriormente, la excitabilidad exploratoria (NS1) se relaciona más y en sentido positivo con la SD, que con la NS: la SD parece representar una metavariable de funcionamiento ejecutivo prefrontal, lo que tiene que ver, por una parte con toma de decisiones, por otra con control emocional y, en último término, con el hallazgo generalizado de una baja capacidad de autodirección relacionada con los trastornos de la personalidad (Pedrero Pérez et al., 2011b; Svrakic et al., 2002). Parece recomendable que las futuras investigaciones consideren por separado la subdimensión NS1.

Comparando estos resultados con los obtenidos en otros contextos culturales y lingüísticos, lo más llamativo resulta ser la notable correlación negativa entre el HA-PE encontrada, de modo similar a lo hallado en población general española (Aluja et al., 2010). Algunos estudios afirman que las diferencias encontradas en el TCI-R en los diferentes países e idiomas podría deberse en parte a diferencias reales en la organización de la personalidad entre culturas (Miettunen, Kantojärvi, Veijola, Järvelin y Joukamaa, 2006); no obstante, el mismo grupo informa de un meta-análisis sobre la administración del TCI en los diferentes países e idiomas (Miet- 
tunen, Lauronen, Kantojärvi, Veijola y Joukamaa, 2008) en el que no se encuentra una variación cultural sistemática respecto a las dimensiones de Cloninger. La posibilidad de que existan diferencias en la organización de la personalidad entre, por ejemplo las culturas mediterráneas frente a las germánicas, medidas a través del TCI o el TCI-R, no es compatible con estos datos.

Nuestros resultados concuerdan con los obtenidos por Pelissolo et al. (2005), observando la tendencia a utilizar la respuesta neutra a medida que avanza el cuestionario, lo que puede ser interpretado como efecto de la fatiga o lasitud creciente frente a un cuestionario tan largo (240 ítems) y no con temas relacionados con el contenido de los ítems. Ninguno de los ítems coincide entre ambos trabajos, pero nuestro estudio también encuentra una ligera, pero significativa, correlación entre el porcentaje de respuestas ' 3 ' ('Igual cierto que Falso') y la numeración del ítem $(r=.18 ; p<.001)$, lo que apunta, como sugieren estos autores, a cierta preferencia por la respuesta neutra a causa de la fatiga. Por ello, una reducción del número total de ítems podría aumentar la consistencia interna y la validez factorial, tanto más si se tuvieran en cuenta los graves problemas detectados en las escalas NS1, RD4 y SD4. Sin embargo, la reducción del número total de ítems, como argumento para obtener un aumento de las propiedades psicométricas, corre el riesgo de reducir la validez predictiva, especialmente para los trastornos clínicos, por lo que todos los intentos de encontrar una forma abreviada del TCI-R deben ser analizados cuidadosamente. En lengua española existen varios intentos en este sentido: el TCI-R-67 (Pedrero Pérez, 2009a), que propone la segregación de la subescala NS1 como escala independiente, y una versión TCI-R-56 (Adan, Serra, Caci y Natale, 2009), basada en estudios previos sobre el TCI (Rigozzi y Rossier, 2004). Con mayor número de ítems, contamos con la versión TCI140 propuesta por Gutiérrez-Zotes et al. (Gutiérrez-Zotes, Cortés, Valero, Peña y Labad, 2005) y que ha sido estudiada también en muestras de adictos (Pedrero Pérez, 2006b).

En definitiva, los resultados obtenidos, en concordancia con otros trabajos previos, ponen en cuestión la validez factorial lineal del TCI-R, en especial para los constructos relacionados con el "temperamento". A pesar de ello, tanto el TCI como el TCI-R han mostrado validez predictiva notable, superando a buena parte de los inventarios de personalidad normal existentes (Grucza y Goldberg, 2007), aunque se requieren estudios que efectúen la misma comparación con otros cuestionarios, más orientados a la evaluación de la personalidad patológica (ej., SNAP, DAPP-BQ, MCMI). Cloninger (2008) respondió a estos problemas sugiriendo que la explicación podría situarse al nivel del comportamiento dinámico no lineal de los circuitos cerebrales retroalimentados de alto nivel, implicando, por ello, y de forma prioritaria, a las dimensiones temperamentales que se presumen con una mayor base biológica. Esta propuesta de rechazo a los análisis lineales en el estudio de la personalidad es compartido por otros autores (p.e., Millon, 1999). Sin embargo, la consecuencia lógica de esta hipótesis debiera haber sido la propuesta y la demanda de métodos específicos de investigación no lineal, aceptables por el autor y su grupo para futuras investigaciones con el TCI-R, haciendo que su hipótesis de no-linealidad fuera empíricamente falsable. No obstante, ninguna propuesta concreta fue explicitada por Cloninger (2008), como han observado también otros autores (Farmer y Goldberg, 2008). Se dispone ya de modelos de análisis no-lineales, que podrían ser de utilidad en el contraste de estas hipótesis (Buckler y Hennig-Thurau, 2008), algo exigible desde el rigor científico.

Limitaciones. El estudio factorial se ha realizado sobre una muestra que cumple el criterio básico de 3 sujetos por variable (Guilford, 1954; Kline, 1986, 1994), si bien otros autores consideran necesario un mínimo de 10 para confirmar la estructura factorial encontrada (Nunnally, 1978; Thorndike, 1982); no obstante, y teniendo en cuenta el abultado número de ítems del cuestionario estudiado, una muestra de más de 800 sujetos pede ser considerada aceptable. También es posible que una buena parte de las deficiencias psicométricas observadas no deban atribuirse únicamente a problemas del instrumento, sino también a diferencias o modificaciones en la estructura de la personalidad de los sujetos de la muestra. El presente trabajo se ha realizado sobre una muestra clínica de adictos a sustancias y, como se ha constatado en otros estudios, es preciso considerar la existencia de un cambio de personalidad (o un cambio transitorio en la estructura de la personalidad), atribuible al estrés o al efecto de las sustancias, que no responde a la esperada estabilidad de los rasgos propuesta por la teoría de Cloninger, entre otras (Pedrero Pérez, 2009c; Pedrero Pérez, López Durán y Olivar Arroyo, 2011a). No obstante, esta limitación es inherente al tema de estudio: la bondad psicométrica del TCI-R en su aplicación a muestras clínicas. También debe considerarse la posibilidad de que, a pesar de haber dejado transcurrir un cierto tiempo (entre 2 y 4 semanas) en el que no se han producido consumos de las sustancias adictivas, éste no sea suficiente para eliminar los efectos residuales del consumo, ni el estrés asociado a la demanda de tratamiento; sin embargo, éste es el tiempo generalmente aceptado para efectuar estudios sobre psicopatología y personalidad (First, Frances y Pincus, 2002; Sonne y Brady, 1998). Otras características que suelen atribuirse a los adictos (ej., impulsividad, impaciencia, inatención) y que en teoría podrían dificultar la cumplimentación de un cuestionario tan largo, son, por una parte, rasgos de personalidad que se pretenden medir, y, por otra, como han mostrado los resultados de este estudio, la tendencia a responder irreflexivamente a medida que se avanza en el cuestionario es estrictamente similar a la encontrada en población general y otras muestras clínicas (Pelissolo et al., 2005).

Como conclusión, debemos destacar que la estructura del TCI-R, en su aplicación a una amplia muestra de personas con adicción a sustancias en tratamiento, ha mostrado importantes debilidades en varias de sus dimensiones principales, en algunas subdimensiones y en un cierto número de ítems que no ratifican una adecuada validez interna y una es- 
tructura factorial satisfactoria. La principal sugerencia derivada del presente estudio tiene que ver con la necesaria reducción de ítems, encaminada a eliminar superposiciones y solapamiento de subescalas, así como a disminuir el cansancio que favorece la emisión de respuestas neutras y reduce la reflexividad ante el estímulo. Pero también sugiere una reflexión de índole teórica: es probable que ni los temperamentos ni los caracteres respondan a las características que la teoría de Cloninger les asignaba inicialmente; ni los primeros parecen tan estables ni los segundos tan independientes de los sustratos biológicos, como ya se ha constatado en algunos trabajos del propio autor (Gillespie, Cloninger, Heath y Martin, 2003). Es más probable que, contra lo que ha defendido la psicología de la personalidad, ésta sea mucho más dinámica de lo que se presuponía, en consonancia con los hallazgos neurocomportamentales de la última década. Posiblemente, los próximos años deban tener en cuenta teorías novedosas que pueden explicar la personalidad como el equilibrio dinámico entre los mecanismos de control superiores y su capacidad para modular los requerimientos de

\section{Referencias}

Adan, A., Serra, J. M., Caci, H. y Natale, V. (2009). A reduced Temperament and Character Inventory (TCI-56). Psychometric properties in a nonclinical sample. Personality and Individual Differences, 46, 687-692.

Aluja, A., Blanch, A, Gallart, S. y Dolcet, J. M. (2010). The Temperament and Character Inventory Revised (TCI-R): Descriptive and factor structure in different age levels. Behavioral Psychology, 18, 385-401.

Alvarez-Moya, E. M., Jiménez-Murcia, S., Aymamí, M. N., Gómez-Peña, M., Granero,, R., Santamaría, J., Menchon, J. M. y Fernández-Aranda, F. (2010). Subtyping study of a pathological gamblers sample. Canadian Journal of Psycbiatry, 55, 498-506.

Álvarez-Moya, E. M., Jiménez-Murcia, S., Granero, R., Vallejo, J., Krug, I., Bulik, C. M. y Fernández-Aranda, F. (2007). Comparison of personality risk factors in bulimia nervosa and pathological gambling. Comprehensive Psychiatry, 48, 452-457.

Arbuckle, J. L. (2005): AMOS 6.0. AMOS Development Corporation, Spring House, PA.

Benjamin, J., Li, L., Patterson, C., Greenberg, B. D., Murphy, D.L. y Hamer, D.H. (1996). Population and familial association between the D4 dopamine receptor gene and measures of novelty seeking. Nature Genetics, 12, 81-84.

Bernstein, I. H. y Teng, G. (1989). Factoring items and factoring scales are different: Spurious evidence for multidimensionality due to item categorization. Psychological Bulletin, 105, 467-477.

Buckler, F. y Hennig-Thurau, T. (2008). Identifying Hidden Structures in Marketing's Structural Models Through Universal Structure Modeling. An Explorative Bayesian Neural Network Complement to LISREL and PLS. Marketing-Journal of Research and Management, 4, 47-66.

Cloninger, C. R. (1986). A unified biosocial theory of personality and its role in the development of anxiety states. Psychiatric Developments, 3, 167-226.

Cloninger, C. R. (1987). A systematic method for clinical description and classification of personality variants. Archives of General Psychiatry, 44, 573-588.

Cloninger, C. R. (1999). The Temperament and Character Inventory-Revised. St Louis, MO: Center for Psychobiology of Personality, Washington University.

Cloninger, C. R. (2003). Completing the psychobiological architecture of human personality development: temperament, character and coherence. En M. Ursula y U. Lindenberger (Eds.), Understanding buman development: dialogues with lifespan psychology (pp.159-181). Dordrecht: Kluwer. centros de orden inferior (Ernst y Fudge, 2009) y que ello explique que, en situaciones en las que el control superior fracasa, los inputs de las estructuras de orden inferior se alternen, traduciéndose en una conducta inestable y ambivalente; ello explicaría el incremento simultáneo, habitualmente observado en muestras de adictos, de dimensiones contrapuestas, como la búsqueda de novedad (p. e., núcleo estriado) y la evitación del daño (p.e., amígdala), acompañado de una puntuación críticamente baja de la autodirección, como metavariable de funcionamiento prefrontal (Ruiz Sánchez de León et al., 2010). Es posible también, que, como sugiere el propio Cloninger, este dinamismo obligue a la utilización de métodos no lineales para el estudio de la relación entre variables que no guardan entre sí una relación en serie, sino que actúan en paralelo. En todo caso, la utilización clínica del TCI$\mathrm{R}$ debe partir del conocimiento de sus limitaciones, haciendo recomendable el uso de las versiones reducidas, alentando la investigación encaminada a su mejora en los próximos años, como ha sido la pauta marcada por el propio autor.

Cloninger, C. R. (2008). The psychobiological theory of temperament and character: Comment on Farmer and Goldberg (2008). Psychological Assessment, 20, 292-299.

Cloninger C. R., Przybeck T. R. y Svrakic, D. M. (1991). The ThreeDimensional Personality Questionnaire: U.S. normative data. Psychological Report, 69, 1047- 1057.

Cloninger, C. R., Przybeck, T. R., Svrakic, D. M. y Wetzel, R. D. (1994). The Temperament and Character Inventory (TCI): $A$ guide to its development and use. St Louis, MO: Center for Psychobiology of Personality, Washington University.

Cloninger, C. R., Svrakic, D. M. y Przybeck, T. R. (1993). A psychobiological model of temperament and character. Archives of General Psychiatry, 50, 975-990.

Cloninger, C. R., Svrakic, N. M. y Svrakic, D. M. (1997). Role of personality self-organization in development of mental order and disorder. Development and Psychopathology, 9, 881-906.

Comings, D. E., Gade-Andavolu, R., Gonzalez, N, Wu, S., Muhleman, D., Blake, H., Mann, M. B., Dietz, G., Saucier, G. y MacMurray, J. P. (2000). A multivariate analisys of 59 candidate genes in personality traits: the temperament and character inventory. Clinical Genetics, 58, 375-385.

Cortés, M. J., Valero, J., Gutiérrez-Zotes, J. A., Hernández, A., Moreno, L., Jariod, M., Martorell, L., Vilella, E. y Labad, A. (2009). Psychopathology and personalitytraits in psychoticpatients and their first-degreerelatives. European Psycbiatry, 24, 476-82.

Cortés Ruiz, M. J., Gutiérrez-Zotes, A., Valero Oyarzábal, J., Jariod Pàmies, M. y Labad Alquézar, A. (2010). Delirios y su relación con el temperamento y carácter en pacientes con trastorno psicótico. Psicothema, 22, 84 91.

Ebstein, R. P., Novick, O., Umansky, R., Priel, B., Osher, Y., Blaine, D., Bennett, E. R., Nemanov, L., Katz, M. y Belmaker, R. H. (1996). Dopamine D4 receptor (DRD4) exon III polymorphism associated with the human trait of novelty seeking. Nature Genetics, 12, 78-80.

Ernst, M. y Fudge, J. L. (2009). A developmental neurobiological model of motivated behavior: Anatomy, connectivity and ontogeny of the triadic nodes. Neuroscience and Biobehavioral Reviews, 33, 367-382.

Farmer, R. F. y Goldberg, L. R. (2008). Brain modules, personality layers, planes of being, spiral structures, and the equally implausible distinction between TCI-R "Temperament" and "Character" scales: Reply to Clonninger (2008). Psychological Assessment, 20 (3), 300-304. 
Fernández Aranda, F, Badia Casanovas, A., Bayón Pérez, C. y Aitken A. (s.f.). Cuestionario de Temperamento y Carácter de Cloninger, versión revisada. Recuperado de http://psychobiology.wustl.edu/joomla/.

Fernández-Aranda, F., Jiménez-Murcia, S., Álvarez-Moya, E. M., Granero, R., Vallejo, J. y Bulik, C. M. (2006). Impulse control disorders in eating disorders: clinical and therapeutic implications. Comprehensive Psychiatry, 47, 482-488.

First, M. B., Frances, A. y Pincus, H. A. (2002). Differential diagnosis step by step. En M. B. First, A. Frances y H. A. Pincus, DSM-IV-TR Handbook of differential diagnosis (pp. 1-14). Washington DC: American Psychiatric Publishing.

Flora, D. B. y Curran, P. J. (2004). An empirical evaluation of alternative methods of estimation for confirmatory factor analysis with ordinal data. Psychological Methods, 9, 466-491.

Fossati, A., Cloninger, C. R., Villa, D., Borroni, S., Grazioli, F., Giarolli, L., Battaglia, M. y Maffei, C. (2007). Reliability and validity of the Italian version of the Temperament and Character Inventory-Revised in an outpatient sample. Comprehensive Psychiatry, 48, 380-387.

Gillespie, N. A., Cloninger, C. R., Heath, A. C. y Martin, N. G. (2003). The genetic and environmental relationship between Cloninger's dimensions of temperament and character. Personality and Individual Differences, 35 , 1931-1946.

Grucza, R. A. y Goldberg, L. R. (2007). The comparative validity of 11 modern personality inventories: predictions of behavioral acts, informant reports, and clinical indicators. Journal of Personality Assessment, 89, 167-187.

Guilford, J. P. (1954). Psychometric Methods. New York: McGraw-Hill.

Gutiérrez, F., Torrens, M., Boget, T., Martín-Santos, R., Sangorrín, J., Pérez, G. y Salamero, M. (2001). Psychometric properties of the Temperament and Character Inventory (TCI) questionnaire in a Spanish psychiatric population. Acta Psychiatrica Scandinavica, 103, 143-147.

Gutiérrez-Zotes, J. A., Bayón, C., Montserrat, C., Valero, J., Labad, A., Cloninger, C. R. y Fernández-Aranda, F. (2004). Inventario del Temperamento y el Carácter-Revisado (TCI-R). Baremación y datos normativos en una muestra de población general. Actas Españolas de Psiquiatría, 32, 8-15.

Gutiérrez-Zotes, J. A., Cortés, M. J., Valero, J., Peña, J. y Labad, A. (2005). Psychometric properties of the abbreviated Spanish version of TCI-R (TCI-140) and its relationship with the Psychopathological Personality Scales (MMPI-2 PSY-5) in patients. Actas Españolas de Psiquiatría, 33, 231-237.

Hansenne, M., Delhez, M. y Cloninger, C. R. (2005). Psychometric properties of the Temperament and Character Inventory-Revised (TCI-R) in a Belgian sample. Journal of Personality Assessment, 85, 40-49.

Hansenne, M., Pinto, E., Pitchot, W., Reggers, J., Scantanburlo, G., Moor, M. y Ansseau, M. (2002). Further evidence on the relationship between dopamine and novelty seeking: a neuroendocrine study. Personality and Individual Differences, 33, 967-977.

Hansenne, M., Pinto, E., Scantanburlo, G., Renard, B., Reggers, J., Fuchs, S., Pitchot, W. y Ansseau, M. (2003). Harm avoidance is related to mismatch negativity (MMN) amplitude in healthy subjects. Personality and Individual Differences, 34, 1039-1048.

Kline, P. (1986). A Handbook of Test Construction. New York: Methuen.

Kline, P. (1994). An Easy Guide to Factor Analysis. Newbury Park: Sage.

Lorenzo-Seva, U. y Ferrando, P. J. (2006). FACTOR: A computer program to fit the exploratory factor analysis model. Behavioral Research Methods, Instruments and Computers, 38, 88-91.

Mardia, K. V. (1970), Measures of multivariate skewnees and kurtosis with applications. Biometrik.a, 57, 519-530.

Martinotti, G., Mandelli, L., Di Nicola, M., Serretti, A., Fossati, A., Borronid, S., Cloninger, C. R. y Janiria, L. (2008). Psychometric characteristic of the Italian version of the Temperament and Character InventoryRevised, personality, psychopathology, and attachment styles. Comprehensive Psycbiatry, 49, 514-522.

Miettunen, J., Kantojärvi, L., Veijola, J., Järvelin, M-R. y Joukamaa, M. (2006). International comparison of Cloninger's temperament dimensions. Personality and Individual Differences, 41, 1515-1526.

Miettunen, J., Lauronen, E., Kantojärvi, L., Veijola, J. y Joukamaa, M. (2008). Inter-correlations between Cloninger's temperament dimensions. A meta-analysis. Psychiatry Research, 160, 106-114.
Millon, T. (1999). Reflections on Psychosynergy: A model for integrating science, theory, classification, assessment, and therapy. Journal of Personality Assessment, 72, 437-456.

Muthén, B. y Kaplan D. (1985). A comparison of some methodologies for the factor analysis of non-normal Likert variables. British Journal of Mathematical and Statistical Psychology, 38, 171-189.

Muthén, B. y Kaplan D. (1992). A comparison of some methodologies for the factor analysis of non-normal Likert variables: A note on the size of the model. British Journal of Mathematical and Statistical Psychology, 45, 1930.

Noble, E. P., Ozkaragoz, T. Z., Ritchie, T. L., Zhang, X., Belin, T. R. y Sparkes, R. S. (1998). D2 and D4 dopamine receptor polymorphisms and personality. American Journal of Medical Genetics, 81, 257-267.

Nunnally, J. C. (1978). Psychometric Theory. New York: McGraw-Hill.

Pedrero Pérez, E. J. (2006a). Temperamento, carácter y trastornos de la personalidad. Aplicación del TCI-R a una muestra de consumidores de drogas en tratamiento y su relación con el MCMI-II. Adicciones, 18, 135148.

Pedrero Pérez, E. J. (2006b). TCI-140: propiedades psicométricas, relación con el TCI-R y con variables de personalidad. Estudio de una muestra de adictos en tratamiento. Trastornos Adictivos, 8, 155-167.

Pedrero Pérez, E. J. (2007). El carácter como predictor de trastornos de la personalidad en adictos: la tipología caracterial de Cloninger y su abordaje psicosocial. Revista Española de Drogodependencias, 32, 342-369.

Pedrero Pérez, E. J. (2009a). TCI-R-67: versión abreviada del TCI-R de Cloninger. Proceso de creación y administración a una muestra de adictos a sustancias en tratamiento. Trastornos Adictivos, 11, 12-23.

Pedrero-Pérez, E. J. (2009b). Evaluación de la impulsividad funcional y disfuncional en adictos a sustancias mediante el inventario de Dickman. Psicothema, 21, 585-591.

Pedrero Pérez, E. J. (2009c). Dimensiones de los trastornos de personalidad en el MCMI-II en adictos a sustancias en tratamiento. Adicciones, 21, 2938.

Pedrero-Pérez, E. J., López-Durán, A. y Olivar-Arroyo, A. (2011a). Addiction: Frontal personality change but not personality disorder comorbidity. Implications for treatment of addictive disorders. In Jordan, M. E. (ed.), Personality Traits Theory, Testing and Influences (pp. 1-36). New York: Nova Publishers.

Pedrero Pérez, E. J. y Rojo Mota, G. (2008). Diferencias de personalidad entre adictos a sustancias y población general. Estudio con el TCI-R de casos clínicos con controles emparejados. Adicciones, 20, 251-261.

Pedrero Pérez, E. J., Ruiz Sánchez de León, J. M., Olivar Arroyo, Á., Rojo Mota, G., Llanero Luque, M. y Puerta García, C. (2011b). Diferencias de personalidad entre adictos al alcohol y controles emparejados: relación con sintomatología frontal y subtipos de adictos. Psicothema, 23, 100-106.

Pelissolo, A., Mallet, L., Baleyte, J. M., Michel, G., Cloninger, C. R., Allilaire, J. F. y Jouvent, R. (2005). The Temperament and Character InventoryRevised (TCI-R): psychometric characteristics of the French version. Acta Psychiatrica Scandinavica, 112, 126-133.

Preiss, M., Kuchařová, J., Novák, T. y Štěpánková, H. (2007). The Temperament and Character Inventory-Revised (TCI-R): A psychometric characteristics of the Czech version. Psychiatria Danubina, 19, 27-34.

Pryor, L. R., Miller, J. D. y Gaughan, E. T. (2009). Testing two alternative pathological personality measures in the assessment of psychopathy: an examination of the SNAP and DAPP-BQ. Journal of Personality Disorders, 23, 85-100.

Rigozzi, C. y Rossier J. (2004). Validation of a short form of the TCI (TCI56) on a sample of young smokers and non-smokers. Annales Médico Psychologiques, 162, 541-548.

Rodríguez, B., Bayón, C., Orgaz, P., Torres, G., Mora, F. y Castelo, B. (2007). Adaptación individual y depresión en una muestra de pacientes oncológicos. Psicooncología, 4, 7-19.

Ruiz Sánchez de León, J. M., Pedrero Pérez, E. J.; Olivar Arroyo, Á., Llanero Luque, M., Rojo Mota, G. y Puerta García, C. (2010). Personalidad y sintomatología frontal en adictos y población no clínica: hacia una neuropsicología de la personalidad. Adicciones, 22, 233-244.

Sato, T., Narita, T., Hirano, S., Kusunoki, K., Goto, M., Sakado, K. y Uehara, T. (2001). Factor validity of the temperament and character inven- 
tory in patients with major depression. Comprehensive Psychiatry, 42, 337341.

Schinka, J. A., Letsch, E. A. y Crawford, F. C. (2002). DRD4 and novelty seeking: results of meta-analyses. American Journal of Medical Genetics, 114, 643-648.

Sonne, S. C. y Brady, K. T. (1998). Diagnosis of personality disorders in cocaine dependent individuals. American Journals on Addictions, 7, 1-6.

Svrakic, D. M., Draganic, S., Hill, K., Bayon, C., Przybeck, T. R. y Cloninger, C. R. (2002). Temperament, character, and personality disorders: etiologic, diagnostic, treatment issues. Acta Psychiatrica Scandinavica, 106, 189195.

Ten Berge, J. M. F. y Kiers, H. A. L. (1991). A numerical approach to the exact and the approximate minimum rank of a covariance matrix. Psychometrike, 56, 309-315.
Thorndike, R. L. (1982). Applied Psychometrics. Boston: Houghton-Mifflin.

Timmerman, M. E. y Lorenzo-Seva, U. (2011). Dimensionality assessmen of ordered polytomous items with parallel analysis. Psychological Methods, 16, 209-20.

Unser, M., Steven, A. C. y Trus, B. L. (1986). Odd men out: a quantitative objective procedure for identifying anomalous members of a set of noisy images of ostensibly identical specimens. Ultramicroscopy, 19, 337 347.

Velicer, W. F. (1976). Determining the number of components from the matrix of partial correlations. Psychometrika, 41, 321-327.

(Artículo recibido: 16-1-2012; revisado: 13-9-2012; aceptado: 16-9-2012) 